\title{
Controlling the unstable emission of a semiconductor laser subject to conventional optical feedback with a filtered feedback branch
}

\author{
I. V. Ermakov ${ }^{1,2 *}$, V. Z. Tronciu ${ }^{3,1}$, Pere Colet $^{1}$ and Claudio R. Mirasso ${ }^{1}$ \\ ${ }^{I}$ Instituto de Física Interdisciplinar y Sistemas Complejos (IFISC, CSIC-UIB), \\ Campus Universitat de les Illes Balears, E-07122 Palma de Mallorca, Spain \\ ${ }^{2}$ Department of Applied Physics and Photonics, Vrije Universiteit Brussel, Pleinlaan 2, 1050 Brussels, Belgium \\ ${ }^{3}$ Weierstrass Institute for Applied Analysis and Stochastics, Mohrenstr. 39, 10117 Berlin, Germany \\ *ilya@ifisc.uib-csic.es
}

\begin{abstract}
We show the advantages of controlling the unstable dynamics of a semiconductor laser subject to conventional optical feedback by means of a second filtered feedback branch. We give an overview of the analytical solutions of the double cavity feedback and show numerically that the region of stabilization is much larger when using a second branch with filtered feedback than when using a conventional feedback one.
\end{abstract}

(C)2009 Optical Society of America

OCIS codes: (190.3100) Instabilities and chaos; (140.5960) Semiconductor lasers

\section{References and links}

1. A. Argyris, D. Syvridis, L. Larger, V. Annovazzi-Lodi, P. Colet, I. Fischer, J. García-Ojalvo, C. R. Mirasso, L. Pesquera, and K. A. Shore, "Chaos-based communications at high bit rates using commercial fibre-optic links," Nature 437(7066), 343-346 (2005).

2. E. Ott, C. Grebogi, and J. A. Yorke, "Controlling chaos," Phys. Rev. Lett. 64(11), 1196-1199 (1990).

3. K. Pyragas, "Continuous control of chaos by self-controlling feedback," Phys. Lett. A 170(6), 421-428 (1992).

4. E. Schöll, and K. Pyragas, "Tunable semiconductor oscillator based on self-control of chaos in the dynamic hall effect," Europhys. Lett. 24(3), 159-164 (1993).

5. C. Lourenço, and A. Babloyantz, "Control of chaos in networks with delay: A model for synchronization of cortical tissue," Neural Comput. 6(6), 1141-1154 (1994).

6. F. R. Ruiz-Oliveras, and A. N. Pisarchik, "Phase-locking phenomenon in a semiconductor laser with external cavities," Opt. Express 14(26), 12859-12867 (2006).

7. H. Erzgräber, D. Lenstra, B. Krauskopf, A. P. Fischer, and G. Vemuri, "Feedback phase sensitivity of a semiconductor laser subject to filtered optical feedback: experiment and theory,” Phys. Rev. E Stat. Nonlin. Soft Matter Phys. 76(2), 026212 (2007)

8. M. Yousefi, D. Lenstra, G. Vemuri, and A. Fischer, "Control of nonlinear dynamics of a semiconductor laser with filtered optical feedback," IEE Proc., Optoelectron. 148(5-6), 233-237 (2001).

9. R. Lang, and K. Kobayashi, "External optical feedback effects on semiconductor injection laser properties," IEEE J. Quantum Electron. 16(3), 347-355 (1980).

10. B. Tromborg, J. H. Osmundsen, and H. Olesen, "Stability analysis for a semiconductor laser in an external cavity," IEEE J. Quantum Electron. 20(9), 1023-1032 (1984).

11. M. Wolfrum, and D. Turaev, "Instabilities of lasers with moderately delayed optical feedback," Opt. Commun. 212(1-3), 127-138 (2002).

12. V. Z. Tronciu, H.-J. Wünsche, M. Wolfrum, and M. Radziunas, "Semiconductor laser under resonant feedback from a Fabry-Perot resonator: Stability of continuous-wave operation," Phys. Rev. E Stat. Nonlin. Soft Matter Phys. 73(4), 046205 (2006).

13. V. Z. Tronciu, I. V. Ermakov, P. Colet, and C. R. Mirasso, "Chaotic dynamics of a semiconductor laser with double cavity feedback: Applications to phase shift keying modulation," Opt. Commun. 281(18), 4747-4752 (2008).

\section{Introduction}

The control of unstable semiconductor lasers (SCL) has received considerable attention during recent years. For example, the presence of periodic or chaotic oscillations can appear when these lasers are subject to conventional optical feedback (COF). Although the chaotic behavior can be useful in, e.g. chaos based applications [1] these oscillations are, in general, unwanted and must be avoided or stabilized. It is our purpose in this work to stabilize periodic

\#106791 - \$15.00 USD Received 26 Jan 2009; revised 20 Apr 2009; accepted 28 Apr 2009; published 11 May 2009 
or chaotic oscillations of a SCL subject to conventional optical feedback into CW emission. To this end we use a second branch subject to a filtered feedback from an external mirror.

One of the pioneering techniques to control unstable periodic orbits was proposed by Ott, Grebogi, Yorke [2]. They showed how to stabilize chaotic oscillations into periodic oscillations by applying small perturbations proportional to the deviation from the desired periodic orbit. Another well known method of control is due to Pyragas [3]. In this method the control is reached by a feedback loop whose round trip time matches the one of the desired periodic orbit. The Pyragas scheme has been successfully applied to different systems [4,5].

The control of a laser subject to COF was studied in ref [6]. It was shown that by using a second COF branch (CFB) and properly adjusting the feedback delays and strengths complex dynamical regimes can be stabilized. However, the control can be achieved only for very precise values of the parameters of the two CFB. To avoid these limitations we propose the use of a filtered optical feedback branch (FFB), as stabilizing branch, in order to get more free parameters and have more opportunities to stabilize the system. We anticipate that when using the FFB the stabilization is easier and much more effective. This control technique would find application in information transmission systems when damages or unavoidable feedback in the transmission channel occur and can result in a behavior that is not desired in the system.

The paper is structured as follows. In Section 2 we describe the model. In Section 3 we show analytical solutions for a COF case and for the case of one branch with COF and the other with FFB. The control using FFB is presented in Section 4, where we highlight the advantages of the proposed setup compared to that of the COF. Finally, the summary and conclusions are given in Section 5.

\section{Setup and the model}

The proposed setup is depicted schematically in Fig. 1. It consists of a SCL coupled to the external reflectors $R_{l}$ and $R_{2}$, that could be implemented, e.g., by using two fiber cavities. One feedback branch, governed by reflectivity $R_{l}$, is the conventional feedback branch (CFB) and the other governed by $R_{2}$ is the FFB.

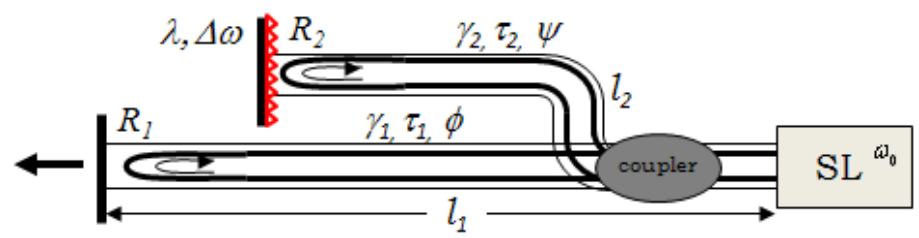

Fig. 1. Investigated setup. A laser with fiber-based external cavities. The cavities lengths are $l_{1}$ $=0.05 \mathrm{~m}$ and $l_{2}=0.03 \mathrm{~m}$. The refractive index of the optical fiber is $\mathrm{n}=1.5$.

Filtered feedback can be obtained from a grating or a Fabry-Perot resonator. In our case we consider a grating filter and, for simplicity, single reflection in both CFB and FFB. The frequency dependent reflectivity in the FFB $r(\omega)$ is described by a Lorenzian function $[7,8]$ with width $\lambda$ and detuning $\Delta \omega$ with respect to the solitary laser frequency $\omega_{0}$.

$$
r(\omega)=\frac{\lambda}{\lambda+i(\Delta \omega-\omega)} .
$$

The laser dynamics is analyzed in the framework of the extended Lang-Kobayashi rate equation [9] for the complex field amplitudes $E(t), F(t)$ and excess carrier density $N(t)$

$$
\begin{gathered}
\dot{E}(t)=\frac{1}{2}(1+i \alpha)\left[g\left(N(t)-N_{0}\right) /\left(1+s|E(t)|^{2}\right)-1 / \tau_{p h}\right] E(t)+\gamma_{1} E\left(t-\tau_{1}\right) e^{i \phi}+\gamma_{2} F(t) . \\
\dot{F}(t)=\lambda E\left(t-\tau_{2}\right) e^{i \psi}+(i \Delta \omega-\lambda) F(t) . \\
\dot{N}(t)=I / e-N(t) / \tau_{n}-g\left(N(t)-N_{0}\right)|E(t)|^{2} /\left(1+s|E(t)|^{2}\right) .
\end{gathered}
$$

\#106791 - \$15.00 USD Received 26 Jan 2009; revised 20 Apr 2009; accepted 28 Apr 2009; published 11 May 2009 
where $\tau_{1}$ and $\tau_{2}$ are the roundtrip times in the CFB and FFB, respectively. $\gamma_{1}$ and $\gamma_{2}$ are the feedback strengths governed by the reflectivity $R_{1}$ and $R_{2}$, respectively. $\phi=\omega_{0} \tau_{1}$ and $\psi=\omega_{0} \tau_{2}$ are the accumulated optical phases in the CFB and FFB, respectively. The second and the third terms in the Eq. (2) correspond to the feedback from CFB and FFB, respectively. The other parameter values are: $\alpha=5$ the linewidth enhancement factor, $g=1.5 \cdot 10^{-5} \mathrm{~ns}^{-1}$ the differential gain, $s=4 \cdot 10^{-7}$ the gain saturation coefficient, $\tau_{p h}=2 \mathrm{ps}$ and $\tau_{n}=2 \mathrm{~ns}$ the photon and carrier lifetimes, respectively; $N_{0}=1.5 \cdot 10^{8}$ the carrier number at the transparency. The injection current is $I=45 \mathrm{~mA}$ (threshold current $I_{t h}=14.7 \mathrm{~mA}$ ).

\section{Stationary states. Saddle-node bifurcation}

The stationary states of the system (2-4) are given by rotating wave solutions (external cavity modes, ECMs) of the form

$$
E(t)=E_{S} \cdot e^{i \omega_{s} t}, F(t)=F_{S} \cdot e^{i \omega_{s} t+i \Phi_{s}}, N(t)=N_{s},
$$

where $E_{s}, F_{s}, \omega_{s}, \Phi_{s}, N_{s}$ are time-independent real constants. Inserting (5) into (2-4) we obtain

$$
\omega_{s}=D_{1} \sin \left(\phi-\omega_{s} \tau_{1}-\arctan (\alpha)\right)+D_{2 e f f} \sin \left(\psi-\omega_{s} \tau_{2}-\arctan (\alpha)+\delta\right),
$$

where $D_{1}=\gamma_{1} \sqrt{1+\alpha^{2}}, D_{2 \text { eff }}=\gamma_{2} \sqrt{1+\alpha^{2}} / \sqrt{1+\left(\left(\omega_{s}-\Delta \omega\right) / \lambda\right)^{2}}, \delta=\arctan \left(\left(\omega_{s}-\Delta \omega\right) / \lambda\right)$.

To simplify the calculations we have neglected the saturation gain, since the qualitative features of the modes location are not affected. Consequently we obtain

$$
N_{s}=\left[1 / \tau_{p h}-2 \gamma_{1} \cos \left(\phi-\omega_{s} \tau_{1}\right)-2 \gamma_{2} \cos (\delta) \cos \left(\psi-\omega_{s} \tau_{2}+\delta\right)\right] / g+N_{0},
$$

For different parameter values Eq. (6) has different number of roots $\omega_{s}$. When only the CFB is present the solutions of Eq. (6) are located on top of an ellipse in the $\left(\omega_{s}, N_{s}\right)$-projection [10]. The COF phase $\phi$ determines the precise location of the points on the ellipse. When the FFB is added the locations of the modes and the general picture become more complicated.
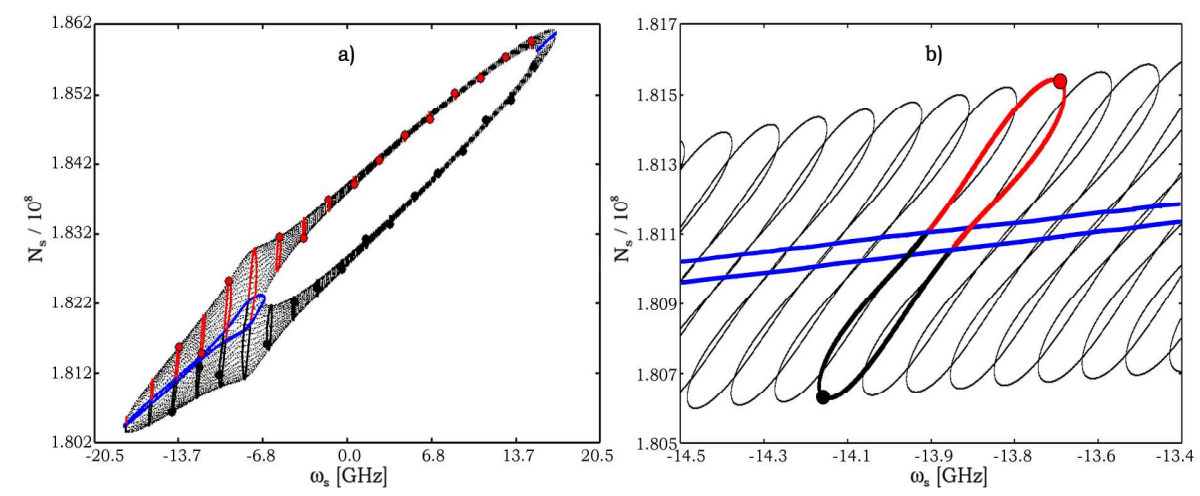

Fig. 2. (a) ECMs in the $\left(\omega_{s}, N_{s}\right)$-plane (Media 1) for $\gamma_{1}=20 \mathrm{~ns}^{-1}, \gamma_{2}=15 \mathrm{~ns}^{-1}, \tau_{1}=0.5 \mathrm{~ns}, \tau_{2}=$ $0.3 \mathrm{~ns}, \lambda=1.6 \mathrm{GHz}$ and $\Delta \omega=-8 \mathrm{GHz}$. (b) zoom of panel (a) around $\omega_{s}=-13.9 \mathrm{GHz}$.

Figure 2(a) shows the typical distribution of the ECMs in the combined CFB and FFB system for the phases $\phi$ and $\psi$ varying in the interval $[0,2 \pi]$. In contrast to the case of single COF our scheme displays a more complicated distribution of modes. Figure 2(b) shows the zoom of Fig. 2(a) in the interval [-14.5 GHz, $-13.4 \mathrm{GHz}$. The stationary states of Eqs. (2-4), for any fixed value of the phase $\phi$, lie on the deformed ellipses (see Figs. 2(a) and 2(b)). Thick black (modes) and red ('anti-modes') lines show the stationary states of the system (2-4) for different values of the phase $\psi$ in the interval $[0,2 \pi]$ and $\phi=0$. When $\phi$ is fixed and $\psi$ is varied the ECMs (big red and black points) move along the deformed black and red thick

\#106791 - \$15.00 USD Received 26 Jan 2009; revised 20 Apr 2009; accepted 28 Apr 2009; published 11 May 2009 (C) 2009 OSA 25 May 2009 / Vol. 17, No. 11 / OPTICS EXPRESS 8751 
ellipses (see Media 1). Big black and red points are modes and 'anti-modes', respectively, when $\phi=\psi=0$. The blue lines show saddle-node bifurcations where modes and anti-modes collide. The 'anti-modes' are always unstable while the modes can be stable or unstable.

By differentiating (6) with respect to $\omega_{s}$, we obtain the condition for saddle-node bifurcations [11,12]. In Fig. 3 we show saddle-node bifurcations in the $\left(\gamma_{2}, \psi\right)$-plane for the laser operating in a periodic (a) and chaotic (b) regimes due to the CFB. The number of modes and anti-modes is shown in colour scale. It can be seen that in the case of the FFB the number of modes decreases when the feedback strength is increased (see red and light blue regions in Fig. 3).
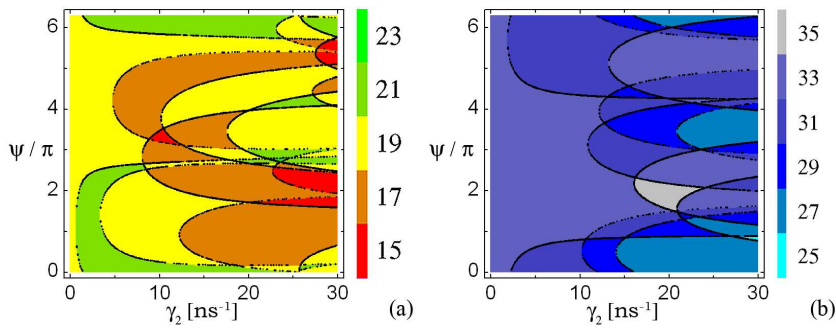

Fig. 3. Lines of saddle-node bifurcation for $\phi=0$, (a) $\gamma_{1}=12 \mathrm{~ns}^{-1}, \Delta \omega=-6 \mathrm{GHz}$ (Media 2) and (b) $\gamma_{1}=20 \mathrm{~ns}^{-1}, \Delta \omega=-8 \mathrm{GHz}$ (Media 3). Other parameters are as in Fig. 2.

\section{Stabilization using filtered feedback}

To study the stabilization process we numerically investigated the system depicted in Fig. 1 under different operating conditions of the CFB. We considered the cases $\gamma_{1}=12 \mathrm{~ns}^{-1}$, when the system develops a periodic behaviour and $\gamma_{1}=20 \mathrm{~ns}^{-1}$ when it operates in a chaotic regime.

Figures 4(a) and 4(b) show the optical spectra in a periodic and chaotic regime. The red line shows the shape of the filter of the FFB whose central frequency is located at the position where the control is optimum.
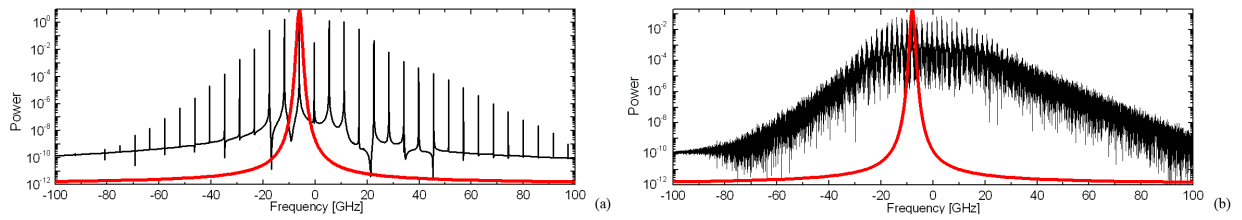

Fig. 4. Optical spectra of a semiconductor laser with a CFB with (a) $\gamma_{1}=12 \mathrm{~ns}^{-1}$ and (b) $\gamma_{1}=20$ $\mathrm{ns}^{-1}$. The other parameters are $\tau_{1}=0.5 \mathrm{~ns}, \phi=0$. The red line shows the shape of the filter with $\lambda=1.6 \mathrm{GHz}$ and (a) $\Delta \omega=-6 \mathrm{GHz}$, (b) $\Delta \omega=-8 \mathrm{GHz}$.

\subsection{Stabilization of periodic oscillations into CW operation}

We begin our analysis by applying a FFB to a system that operates in a periodic regime due to a CFB. Figures 5(a)-5(c) show the regions (light blue) in the parameter space where control is achieved by using the FFB with $\tau_{2}=0.3 \mathrm{~ns}$ and $\Delta \omega=-6 \mathrm{GHz}$. Since the filter width strongly influences the dynamics of the laser the system exhibits different opportunities of control. We observed that the size of the regions where control can be achieved increases with $\gamma_{2}$ (see Figs. 5(a)-5(c) and Medias 4-6). For values of $\gamma_{2}>\gamma_{1}$ the regions where the control is possible covers up to $50 \%$ of the $(\psi, \lambda)$-parameter space (see Fig. 5 (c)).

Figures 5(d)-5(f) show the role of the detuning between the central frequency of the filter and the solitary laser frequency for $\psi=2$. In fact, control is only possible for specific values of the filter detuning. We also noted that the control is very sensitive to the CFB phase since by changing it we shift the peaks of the optical spectrum (see Media 7). The dependence of 
the control regions on the phase is reduced when $\gamma_{2}$ is increased (see Figs. 5(d)-5(f), Media 7 , 8,9 ), but in general the larger filter width $\lambda$ is the smaller the stabilization regions are.
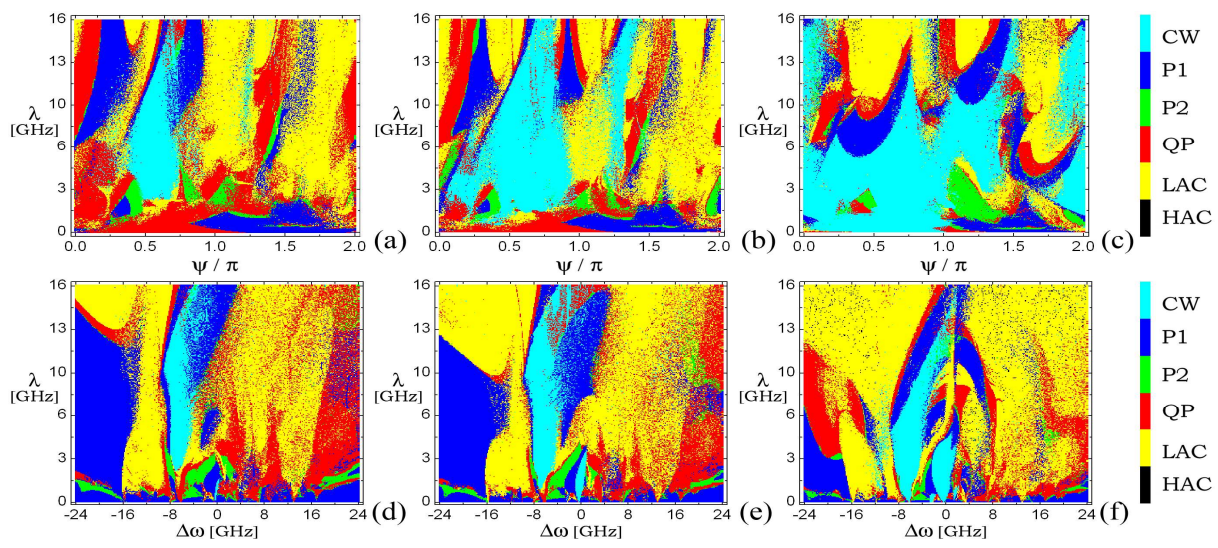

Fig. 5. Control domain of the CFB with $\tau_{1}=0.5 \mathrm{~ns}, \gamma_{1}=12 \mathrm{~ns}^{-1}$ and $\phi=0$ for (a), (d) $\gamma_{2}=8 \mathrm{~ns}^{-1}$ (Media 4, Media 7); (b), (e) $\gamma_{2}=10 \mathrm{~ns}^{-1}$ (Media 5, Media 8) and (c), (f) $\gamma_{2}=15 \mathrm{~ns}^{-1}$ (Media 6, Media 9). The notations are: CW - continuous wave; P1, P2 - period one and two oscillations respectively; QP - quasi-periodic oscillation; LAC, HAC - low and high amplitude chaos respectively.
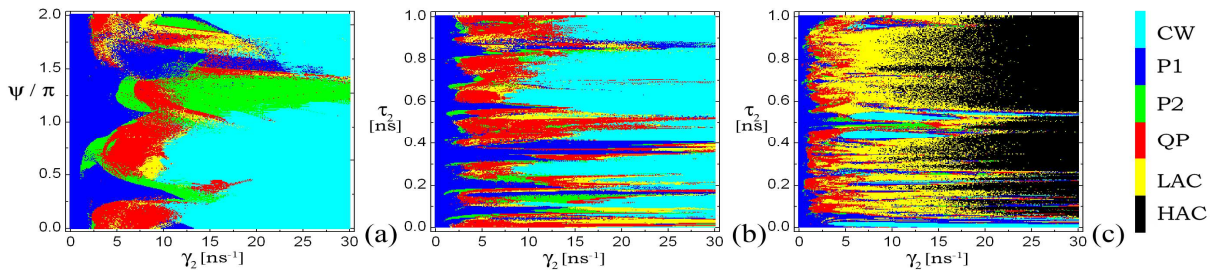

Fig. 6. Effect of control of CFB. Parameters are (a) $\tau_{2}=0.3 \mathrm{~ns}, \lambda=1.6 \mathrm{GHz}$ (Media 10); (b) $\psi$ $=2, \lambda=1.6 \mathrm{GHz}$ (Media 11); (c) $\psi=2, \lambda=\infty$ (Media 12). Other parameters are as in Fig. 4(a).

Figure 6(a) shows the parameter regions where the control can be achieved as a function of $\gamma_{2}$. Large $\gamma_{2}$ values stabilize the system while the number of ECMs in Fig. 3(a) decreases. Figures 6(b) and 6(c) show the influences of the time delay in the dynamics when using narrow filters. and a CFB, $\lambda=\infty$, respectively. It is clear that the parameter regions in which $\mathrm{CW}$ operation is stabilized are much broader when using the FFB. It is worth mentioning that the system is quite sensitive to phase shifts in both cases (Media 11, 12).

\subsection{Stabilization of chaotic fluctuations into CW operation}

In this section we show that with help of a FFB chaotic oscillations can be also suppressed. Figure 7 shows the regions of stabilization for different parameters, when the COF branch is operating in a chaotic regime. An increase of $\gamma_{2}$, as shown in Fig. 7 and (Media 13, 14, 15, 16, 17, 18), leads to the widening of CW regions. Figures 7(a)-7(c) show the control regions (light blue) for $\tau_{2}=0.3 \mathrm{~ns}$ and $\Delta \omega=-8 \mathrm{GHz}$. The filter width $\lambda$ and the detuning $\Delta \omega$ still play a decisive role. Particularly important is the detuning as shown in Figs. 7(d)-7(f) for $\psi=5$. For $\Delta \omega \approx-8 \mathrm{GHz}$ and $\Delta \omega \approx-15 \mathrm{GHz}$ stabilization can easily be obtained while for other values of the detuning the regions of stabilization are practically absent, except for extremely large $\gamma_{2}$.

In the following we show in more detail the regions of control of $\mathrm{CW}$ operation (light blue) when the feedback strength $\gamma_{2}$ is increased. Figure 8(a) shows these regions of control in 

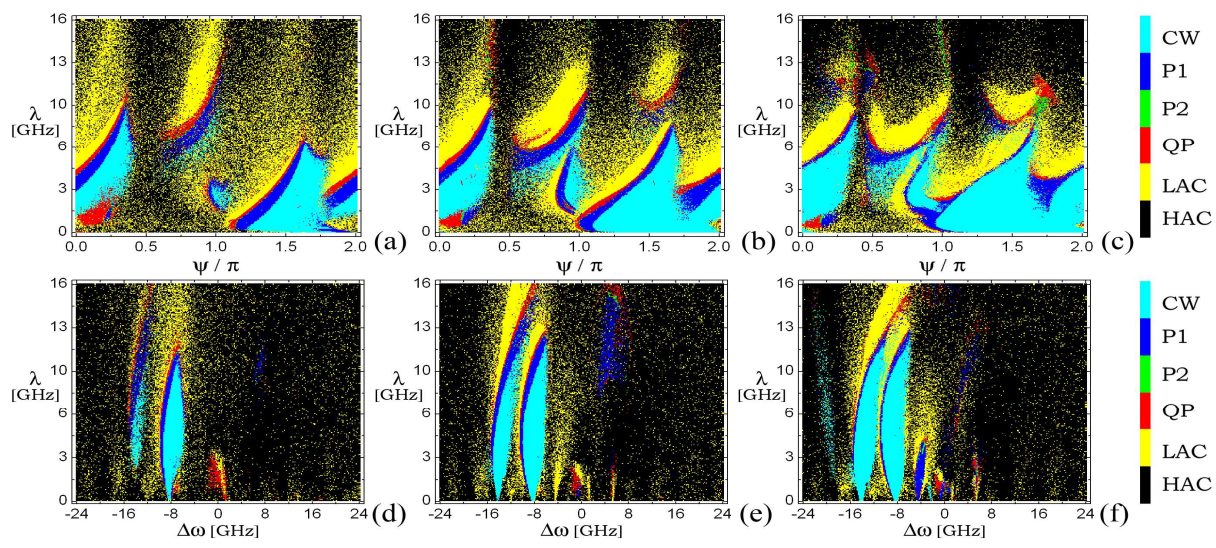

Fig. 7. Control domain of CFB with $\tau_{1}=0.5 \mathrm{~ns}, \gamma_{1}=20 \mathrm{~ns}^{-1}$ and $\phi=0$ for (a), (d) $\gamma_{2}=15 \mathrm{~ns}^{-1}$ (Media 13, Media 16); (b), (e) $\gamma_{2}=20 \mathrm{~ns}^{-1}$ (Media 14, Media 17) and (c), (f) $\gamma_{2}=25 \mathrm{~ns}^{-1}$ (Media 15, Media 18).
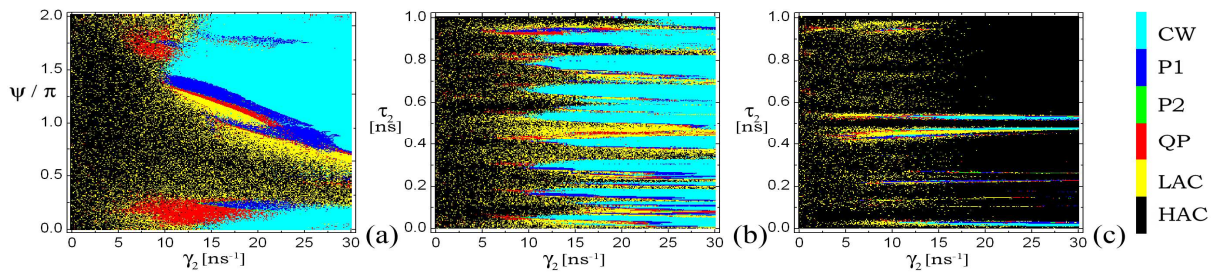

Fig. 8. Effect of control of CFB. Parameters are (a) $\tau_{2}=0.3 \mathrm{~ns}, \lambda=1.6 \mathrm{GHz}$ (Media 19); (b) $\psi$ $=5, \lambda=1.6 \mathrm{GHz}$ (Media 20); (c) $\psi=5, \lambda=\infty$ (Media 21). Other parameters are as in Fig. 4(b).

$\left(\gamma_{2}, \psi\right)$-plane. Figures 8(b) and 8(c) (Media 20 and 21) show an evident advantage of the control in a FFB system compared to that performed by a second CFB.

\section{Summary and conclusions}

We have shown that stabilization of a chaotic SCL to CW operation using a FFB is more effective than a second CFB. The main advantage of FFB system compared to CFB one is that the former has broader CW regions of control and stabilization can be easily achieved. Also stabilization regions depend less on delay time and feedback strengths ratio.

The physical insight behind this fact is that the filtered feedback allows to selectively reinject a specific frequency range, as shown in Fig. 4. In this way, CW instabilities originated from a Hopf bifurcation can be suppressed by feeding-back the Hopf frequency, with an appropriate feedback phase, leading to a destructive interference. As shown in Fig. 5 only some phases lead to CW stabilization. By using a CFB it is also possible to induce destructive interference on that frequency, however, since all the other frequencies are fed-back as well the reinjected light may easily induce an effective reduction of the threshold of other instabilities. This effect can be seen, for example, in Fig. 5 where the $\mathrm{CW}$ stable region decreases, while more chaotic regions appear, as the filter width $\lambda$ is increased. In fact, we have recently shown that CFB systems exhibit strong chaos for increasing feedback strength in one cavity [13]. It is worth mentioning that if the filter width is too narrow and it is not well tuned at the Hopf frequency, the FFB is inefficient. For a practical implementation one should choose $\lambda \sim 3$ to $5 \mathrm{GHz}$.

From a more mathematical point of view, the decrease of the number of ECMs when the feedback strength is high is responsible for the stabilization to occur. If instead a second CFB branch is added to a laser with a CFB the number of ECMs increases and the stabilization is 
not as effective as with FFB. The results obtained could also be interpreted by the phase locking phenomenon described in detail in [6].

\section{Acknowledgments}

Authors acknowledge financial support from the EC project PICASSO, IST-2005-34551 and MICINN and FEDER under grants TEC2006-10009 (PhoDeCC), TEC-2006-28105-E and FIS2007-60327 (FISICOS). IVE acknowledges the Russian President Stipend and support from the Belgian Science Policy Office under grant No. IAP-VI10 as well as from the Research Foundation-Flanders (FWO).

\#106791 - \$15.00 USD Received 26 Jan 2009; revised 20 Apr 2009; accepted 28 Apr 2009; published 11 May 2009 (C) 2009 OSA 